\title{
THE CALCULUS OF VARIATIONS APPLIED TO NÖRLUND'S SUM*
}

BY TOMLINSON FORT

Nörlund's sum† function

$$
\int_{c}^{u} f(x) \Delta x
$$

has many resemblances to a definite integral. The purpose of the present note is to point out how some of the classical methods of the calculus of variations can be applied to such a sum. It may be that the field will prove fruitful for further research.

We shall consider the problem of minimizing (maximizing) the sum

$$
\int_{c}^{b} F\left(x, y, \Delta y, \Delta^{2} y, \cdots, \Delta^{n} y\right) \Delta x,
$$

where we have exactly the same understanding of what constitutes a minimum as in the classical problem of the definite integral.

1. Euler's Equation. We shall seek a necessary condition that a continuous real $y$ minimize

$$
\int_{c}^{b} F(x, y, \Delta y) \Delta x
$$

similar to Euler's equation for the corresponding integral. The condition of fixed end points in the integral problem is here replaced by the condition that $y$ be fixed over the interval $c \leqq x \leqq c+1$ and at the point $b$.

For brevity in writing denote $\Delta y$ by $y^{\prime}, F(x, y, \Delta y)$ by $F(x)$, and assume that $y$ is continuous and that $F, F_{y}$, and $F_{y^{\prime}}$ are continuous in their arguments throughout all neighborhoods considered in the sequel.

* Presented to the Society, September 9, 1937.

$\dagger$ Milne-Thompson, The Calculus of Finite Differences, page 201. In the present paper the difference interval is assumed to be 1 . 
Assume that $y$ minimizes (2). Give to $y$ a variation $\epsilon \eta(x)$ where $\eta(x)=0$ when $x \leqq c+1$, when $x=b$, and when $x>M$, where $M$ equals $b$ plus an integer. We assume also that $\eta(x)$ is continuous throughout its range of definition. Let $\Delta \eta=\eta^{\prime}$.

Under these restrictions

$$
\phi(\epsilon)=\int_{c}^{b} F\left(x, y+\epsilon \eta, y^{\prime}+\epsilon \eta^{\prime}\right) \Delta x
$$

converges and is a function of $\epsilon$.

We can differentiate under the integral sign inasmuch as all that is involved is an integral over a finite interval and a finite sum. We thus obtain the formula

$$
\begin{aligned}
\left.\frac{d}{d \epsilon} \phi(\epsilon)\right|_{\epsilon=0} & =\int_{c}^{b}\left(\eta F_{y}+\eta^{\prime} F_{y^{\prime}}\right) \Delta x \\
& =\int_{c}^{b} \eta F_{y} \Delta x+\int_{c}^{b} \eta^{\prime} F_{y^{\prime}} \Delta x .
\end{aligned}
$$

Summation by parts* yields

$$
\begin{aligned}
\int_{c}^{b} \eta^{\prime}(x) F_{y^{\prime}} \Delta x & =F_{y^{\prime}}(b) \int_{c}^{b} \eta^{\prime}(x) \Delta x-\int_{c}^{c+1} F_{y^{\prime}}(x) \int_{c}^{x} \eta^{\prime}(x) \Delta x d x \\
& -\int_{c}^{b} \Delta F_{y^{\prime}}(x) \int_{c}^{x+1} \eta^{\prime}(x) \Delta x \Delta x \\
& =F_{y^{\prime}}(b) \eta(b)-\int_{c}^{c+1} F_{y^{\prime}}(x) \eta(x) d x \\
& -\int_{c}^{b} \eta(x+1) \Delta F_{y^{\prime}}(x) \Delta x \\
& =-\int_{c}^{b} \eta(x+1) \Delta F_{y^{\prime}}(x) \Delta x \\
& =-\int_{c+1}^{b+1} \eta(x) \Delta F_{y^{\prime}}(x-1) \Delta x \\
& =-\int_{c+1}^{b} \eta(x) \Delta F_{y^{\prime}}(x-1) \Delta x \text { since } \eta(b)=0 .
\end{aligned}
$$

* Milne-Thompson, page 206. 
Moreover, since $\eta(x)=0$ also when $c \leqq x \leqq c+1$,

Consequently

$$
\int_{c}^{b} \eta(x) F_{y}(x) \Delta x=\int_{c+1}^{b} \eta(x) F_{y}(x) \Delta x .
$$

$$
\left.\frac{d \phi}{d \epsilon}\right|_{\epsilon=0}=\int_{c+1}^{b} \eta(x)\left[F_{y}(x)-\Delta F_{y^{\prime}}(x-1)\right] \Delta x .
$$

This is necessarily zero for a minimum. Then necessarily

$$
F_{y}(x)-\Delta F_{y^{\prime}}(x-1)=0, \quad x \geqq c+1 .
$$

Suppose it were not true at $\bar{x} \geqq c+1$ where $\bar{x} \neq b+n,(n=0,1$, $2, \cdots)$. Assume $\eta=0$ at all points except a neighborhood of $\bar{x}$ not extending to any $b+n$, and throughout which $F_{y}(x)$ $-\Delta F_{y^{\prime}}(x-1)$ retains the same sign. For an $\eta \neq 0$ throughout this neighborhood this yields $d \phi /\left.d \epsilon\right|_{\epsilon=0} \neq 0$. Moreover, since $y$ is continuous it must also satisfy (3) when $x=b+n$; also $M$ is as large as we please. Hence, a necessary condition that a continuous $y$ render (2) a minimum (maximum) is the satisfaction of (3), which we write

$$
F_{y}(x+1)-\Delta F_{y^{\prime}}(x)=0, \quad x \geqq c .
$$

The resemblance of this to Euler's equation for the integral is immediate.*

By exactly similar arguments making the assumptions that $\eta(x)=0, c \leqq x \leqq c+n$, and $\eta(b)=\eta(b+1)=\cdots=\eta(b+n-1)=0$, we arrive at the following analogue for Euler's equation as a necessary condition for the rendering of (1) a minimum (maximum):

$F_{y}(x+n)-\Delta F_{y^{\prime}}(x+n-1)+\cdots+(-1)^{n} \Delta^{n} F_{y^{(n)}}(x)=0, \quad x \geqq c$, where $y^{(n)}=\Delta^{n} y$.

\section{LEHIGH UNIVERSITY}

* Some special cases of the difference equation (4) were studied in detail by the author in the American Mathematical Monthly of March 1936. In that paper the independent variable was limited to integral values. Necessary modifications to make the discussions given there apply when the independent variable is continuous are not considered of sufficient interest to warrant printing. 